\title{
Unterstützungswerkzeuge für Entwickler von graphischen Benutzungsoberflächen
}

\author{
Frank Bachmann, Michael Porschen, Manfred Ramm, \\ Harald Reiterer, Stefan Schäfer, Helmut Simm \\ Gesellschaft für Mathematik und Datenverarbeitung, Universitait Wien
}

\section{Zusammenfassung}

Durch den Einsatz von rechnerbasierten Unterstützungswerkzeugen, die in ein Entwicklungswerkzeug für graphische Benutzungsobertlichen integriert sind, werden Entwickler in Fragestellungen der soltware-ergonomischen Gestaltung unterstützt. Daunit wird den Entwicklem die Möglichkeit eines hedartsorientierten Erlernens und Anwendens von Ergonomiewissen geboten. Die verschiedenen Unterstülzungswerkzeuge machen von objekt-orientierten, multimedialen und wissensbasierten Techniken Gehrauch, um das Ergonomiewissen zu vermitteln.

\section{Einleitung}

Im folgenden werden Ergehnisse des Gemeinschaftsprojektes "User Intertace Design Assistance (IDA)" der GMD und der FHD Darmstadt vorgestellt [25]. Ziel des Vorhabens ist es, Entwicklem von gruphischen Benutzungsobertlïchen rechnerbasierte Unterstïtzungswerkzeuge zur Verfïigung zu stellen, die sie bei der Umsetzung von software-ergonomischem Gestaltungswissen unterstützen. Anknüpfungspunkte für die Durchführung des Projektes waren einerseits die zunehmende Bedeutung von softwareergonomischen Forderungen aufgrund der von der EU-Kommission verabschiedeten "Richtlinie üher die Mindestvorschriften heziiglich der Sicherheit und des Gesundheitsschutzes bei der Arheit an Bildschirmgeriten [8]" und andererseits empirische Untersuchungen, welche die unzureichende Verbreitung software-ergonomischer Elkenntnisse in der Praxis festgestellt hahen $[4,19]$. Diese Untersuchungen zeigten, daß ein hoher Prozentsatz der Softwareentwickler über keinerlei oder nur geringfügige software-ergonomische Kenntnisse vertügen. Zur Überwindung dieses Detizits wurde von der üherwiegenden Mehrheit der befragten Entwickler eine rechnerbasierte Vermittlung 
software-ergonomischer Gestaltungsanforderungen, während des Entwicklungsprozesses, präferiert. Dazu henötigen die Entwickler leistungsfähige Entwicklungswerkzeuge (z.B. User Interface Management Systems, UIMS), die sie im EntwicklungsprozeB unterstiitzen und sie gleichzeitig bei der Einhaltung der software-ergonomischen Anforderungen anleiten.

Durch die im Rahmen des hier vorgestellten Projektes realisierten und in ein UIMS integrierten Unterstützungswerkzeuge soll erreicht werden, dals Entwickler

- software-ergonomische Kenntnisse während des Implementierungsprozesses, gleichzeilig mit der Handhahung des Entwicklungswerkzeuges, vermittelt hekommen (learning on demand),

- vorgefertigte, ergonomischen Anforderungen entsprechende Dialoghausteine einer Benutzungsoberlläiche verwenden können (reusability),

- hei der Anwendung von ergonomischen Standards und Richtlinien angeleitet werden (usability) und

- die ergonomische Qualität der entworfenen Benutzungsobertlüchen schon im Entwicklungsprozefo üherprüfen lassen können (quality assurance).

\section{Vorgehensweise bei der Entwicklung der Unterstützungs- werkzeuge}

Es war nicht Ziel des Vorhahens ein neues Entwicklungswerkzeug für graphische Benutzungsoherflächen zu entwickeln, sondern marktgängige mit zusätzlichen Unterstützungsleistungen auszustatten. Dazu komnte ein Hersteller eines UIMS (ISA GmbH, Stuttgart) als Kooperationspartner gewonnen werden. Von diesem wurden hestimmte Erweiterungen an dem UIMS vorgenommen sowie definierte Schnittstellen offengelegt. Dies waren unahdinghare Voraussetzungen für eine Integration der verschiedenen Unterstützungswerkzeuge.

Damit konkrete Anforderungen an die Unterstützungswerkzeuge erlaßßt und sie im praktischen Einsatz evaluiert werden konnten, wurden sie für ausgewählte Anwendungsdomänen entwickelt. Dazu wurden eine Reihe von Industriekontakten geknüpft und durch Workshops, Firmenhesuchen, Analysen vor Ort etc. vertieft. Diese Industriekontakte führten zu Kooperationsprojekte mit einigen Industriefirmen (Software AG, Darmstadt; SAP AG, Walldort/Baden; Hoechst AG, Frankfurt/Main). Im Ruhmen dieser Kooperationsprojekte wurden die verschiedenen Unterstützungswerkzeuge auf PCs (DOS/Windows) und Workstations (UNIX/Motif) prototypisch realisiert. Die umfussende Evaluierung dieser Prototypen im praktischen Einsatz wird derzeit durchgeführt. Die dabei gewonnen Erkenntnisse hilden die Grundlage füir eine evolutionäre Weiterentwicklung der Unterstützungswerkzeuge. 
Als Quelle zur Ableitung des software-ergonomischen Wissens wurden Gestaltungsanforderungen aus Normen (z.B. [12]), Style Guides (z.B. [11, 18, 21]) und herstellerunabhångigen Gestaltungsrichtlinien (z.B. $[14,17])$ entnommen.

\section{Typischer Einsatz der Unterstützungswerkzeuge}

Abb. 1 zeigt eine typische Einsatzsituation für die verschiedenen Unterstiitzungswerkzeuge. Die getrennte Pråsentation der DA Unterstiitzungswerkzeuge und des UIMS ist der prototypischen Realisierung geschuldet. Die enge Kooperation mit dem Herstellern des UIMS soll es später ermöglichen, die verschiedenen Leistungen der Unterstïtzungswerkzeuge in das UIMS zu integrieren.

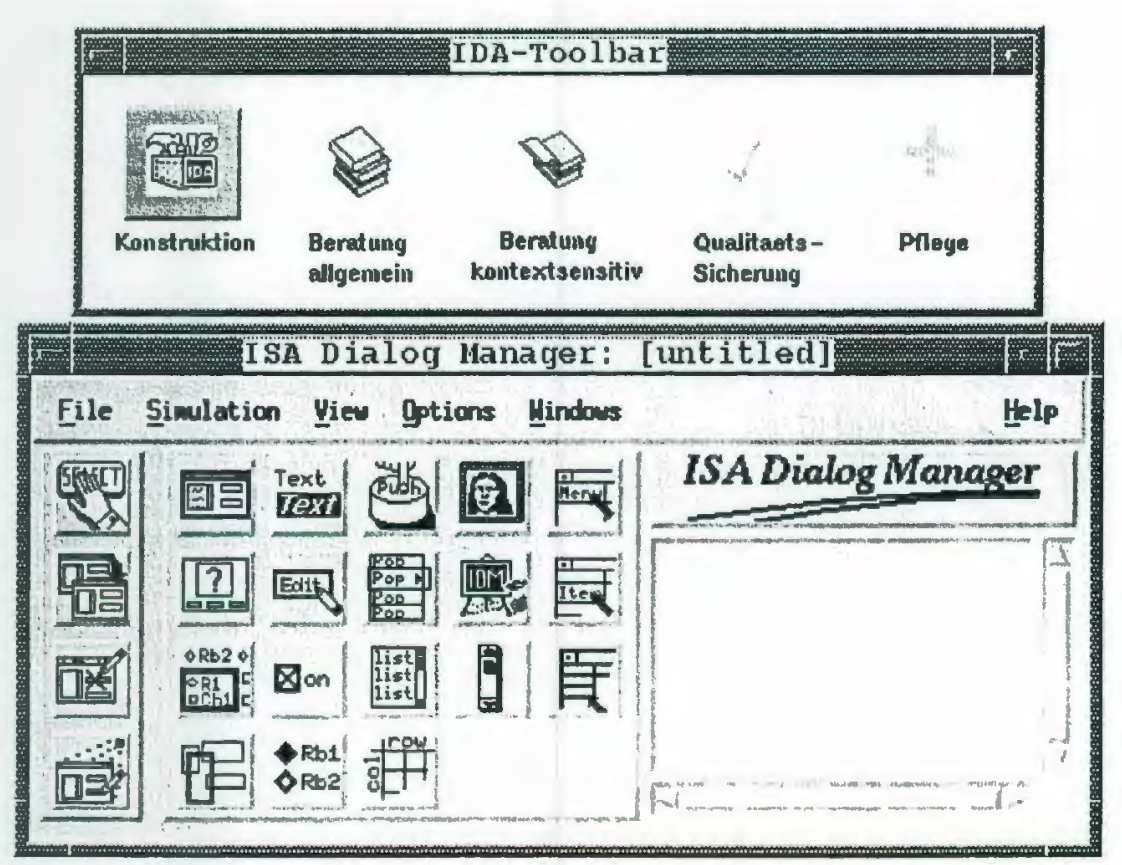

Abb. 1:

$$
\begin{gathered}
\text { Typise } \\
\text { he } \\
\text { Einsat } \\
\text { zsiluat } \\
\text { ion der } \\
\text { Inter- } \\
\text { stützu } \\
\text { ngs- } \\
\text { werkze } \\
\text { uge }
\end{gathered}
$$

Ein Entwickler implementiert unter Verwendung eines UIMS - eine graphische Benutzungsobertläche einer Softwareapplikation. Dazu nutzt er eine

Klassenhibliothek mit vorgefertigten Dialoghausteinen. Zugang zu dieser Bibliothek bietet ihm das Unterstützungswerkzeug Konstruktion, das über ein Icon in der "IDAToolbar" aktivierbar ist [23]. Die Bibliothek hat für den Entwickler eine Art "Baukastenfunktion", indem ihm vorgefertigte Klassen von Dialogbausteine zur Verfüigung gestellt werden (reusability). Diese Dialoghausteine entsprechen software-ergonomischen Gestaltungsforderungen (siehe Abschnitt 2) und enthalten einfache und komplexe Dialogobjekte (z.B. Push Button, Tabelle, Dialogfenster), sowie typische Dialogabläufe (z.B. Hilfe, Fehler, Auswahldialoge). Dialnghausteine kionnen auch komplette, 
anwendungsspezifïsche Fensterarchitekturen repräsentieren (z.B. Hauptfenster mit Tochterfenstern und Dialogfenstern), die auf einem der gängigen Architekturkonzepten basieren (z.B. Single Document Interface). Durch die Anwendung von ergonomischen Dialoghausteine im Entwicklungsprozeß wird sowohl eine konsistente als auch eine aufgabenangemessene und benutzerfreundliche Realisierung der Benutzungsobertläche sichergestellt (usability). Eine wichtiges Kriterium für den Erfolg der Bibliothek stellt die Zugänglíchkeit zu den vordefinierten Dialogbausteinen dar. Die Zugänglichkeit beeinflußt maßgehlich die Nützlichkeit tür den Entwickler. Durch verschiedene Navigations- und Prüsentationsinstrumente (z.B. Browser, Suchfunktionen, Animationen) wird der Entwickler heim Finden geeigneter Dialoghausteine unterstützt. Kommt der Entwickler zur Erkenntnis, daß3 der gefundene Dialogbausteine seinen Wünschen entspricht, kann er eine Instanz der Objektklasse erzeugen.

Ist dem Entwickler beispielsweise der Verwendungszweck eines ausgewählten Dialogbausteines nicht klar, kann er die online verfüghare multimediale kontextsensitive Beratung aktivieren, die ebenfalls mittels eines Icons in der "IDA-Toolbar" aktivierbar ist. Die online-Beratung setzt zur Wissenspräsentation Texte, Graphiken, Animationen, Sprache und interaktive Beispiele ein und bietet vielfältige Navigationstechniken. Neben dem Verwendungszweck von vorgefertigten Dialoghausteinen kann sich der Entwickler auch in anschaulicher Weise allgemeines ergonomisches Wissen zur Implementierung von graphischen Benutzungsoberflächen vermittelt lassen. Der Einstieg in die allgemeine Beratung erfolgt in diesem Fall durch Aktivieren des Icons "Beratung allgemein" in der "IDA-Toolbar". Im Kapitel 4 erfolgt eine austïihrliche Darstellung der verwendeten Konzepte und Techniken der online-Beratung.

Möchte der Entwickler seine bisherigen Implementierungen einer automatischen software-ergonomischen Qualitåtssicherung unterziehen, kann er die, auf wissenshasierten Techniken beruhende, Qualirätssicherung über die "IDA-Toolbar" aktivieren [26]. Diese wertet Entwicklungsergehnisse aufgrund einer expliziten Anforderung durch den Entwickler aus (software quality assurance). Das Ergonomiewissen der verschiedenen Quellen (siehe Abschnitt 2) wurde mittels Regeln in die Wissenshasis aufgenommen. Nach Aktivierung der Qualitätssicherung werden die Daten der Benutzungsobertläche ahgespeichert und in die Qualitätssicherung eingelesen. Anschließend werden automatisch die Inferenzmechanismen aktiviert, die das Abarheiten der Regeln über die Ohjekte der Benutzungsobertläche steuern. Nach der Regelabarbeitung erhält der Entwickler Informationen zu den gefundenen ergonomischen Abweichungen. Zur weiteren Bearbeitung hat der Entwickler mehrere Aktionen zur Auswahl. Er kann sowohl in die Beratung als auch in die Konstruktion verzweigen, um Hinweise zur Fehlerkorrektur zu erhalten. Bei bestimmten Objekten kann auch in der Qualitätssicherung eine automatische Korrektur vorgenommen werden. 
Ergonomiewissen unterliegt einem stetigen Wandel aufgrund neuer wissenschaftlicher Erkenntnisse und des technischen Fortschritts. Damit ist die Notwendigkeit offensichtlich, Möglichkeiten zur Pflege des in den IDA-Unterstützungswerkzeugen vorhandenen Ergonomiewissens bereitzustellen. Dazu wird ein weiteres Unterstützungswerkzeug angeboten, das über die "IDA-Toolbar" mittels des Pllegeicons aktiviert werden kann. Zum einen ist das in jedem einzelnen Unterstuitzungswerkzeug vorhandene Wissen zu pflegen, zum anderen ist die Konsistenz zwischen den Wissensbeständen der einzelnen Unterstützungswerkzeuge zu gewährleisten.

\section{Vermittlung ergonomischen Wissens mittels hypermedialer Techniken}

Die IDA-Beratung wendet sich an Entwickler von Benutzungsobertlächen, die bislang wenig Erfahrung in ergonomischen Design von Benutzungsobertlächen hesitzen. Es soll aber auch Experten ein Nachschlagewerk zur Verfïigung gestellt werden. Die Beratung verfolgt vor allem tutorielle Zwecke. Kurzfristig soll fehlendes ergonomisches Wissen, während des Entwurfprozesses der Benutzungsoherflïche, hedarfsorientiert vermittelt werden (learning on demand). Längerfristig wird ein permanenter Wissenstransfer angestrebt, so dal.3 die Konsultation der Beratung immer seltener erfolgen muß. Mit diesen Zielsetzungen mul3 sich die IDA-Beratung der Konkurrenz konventioneller Methoden der Wissensgewinnung (z. B. Lesen von Style Guides, Befragen von Experten) stellen. Das Beratungssystem erweitert durch den Einsatz von multimedialen Techniken die Möglichkeiten verfügbarer online-Hilfesysteme (z.B. Animationen, Videos, Ton, Sprache). Die multimediale IDA-Beratung muls die Akzeptanz durch die Entwickler mit Hilfe einer konsequenten Ausnutzung der Vorteile der rechnerbasierten Realisierung erreichen. Dieser Anspruch diente als Leitlinie füir die Implementierung und schlägt sich in drei grundlegenden Gestaltungsempfehlungen nieder [22]:

- Verwendung von möglichst wenig Text,

- Darstellung von Abläufen und Vorgängen durch dynamische Medien,

- interaktive Einbindung des Entwicklers.

\subsection{Strukturierung der Inhalte}

Zur Strukturierung der Inhalte der IDA-Beratung, deren Quellen hereits in Abschnitt 2 genannt wurden, boten sich zwei Altemativen an: eine hierarchische oder eine netzwerkartige Struktur. Da das Ergonomiewissen aus hierarchisch strukturierten Büchern entnommen wurde, fiel die Entscheidung zugunsten einer hierarchischen Struktur als primåre Strukturierung. Damit sollen den Entwicklern ein Anknüpien an vertraute Me- 
dien der Wissensvermittlung erleichtert werden. Abb. 2 zeigt einen Ausschnitt dieser Inhaltsstruktur.

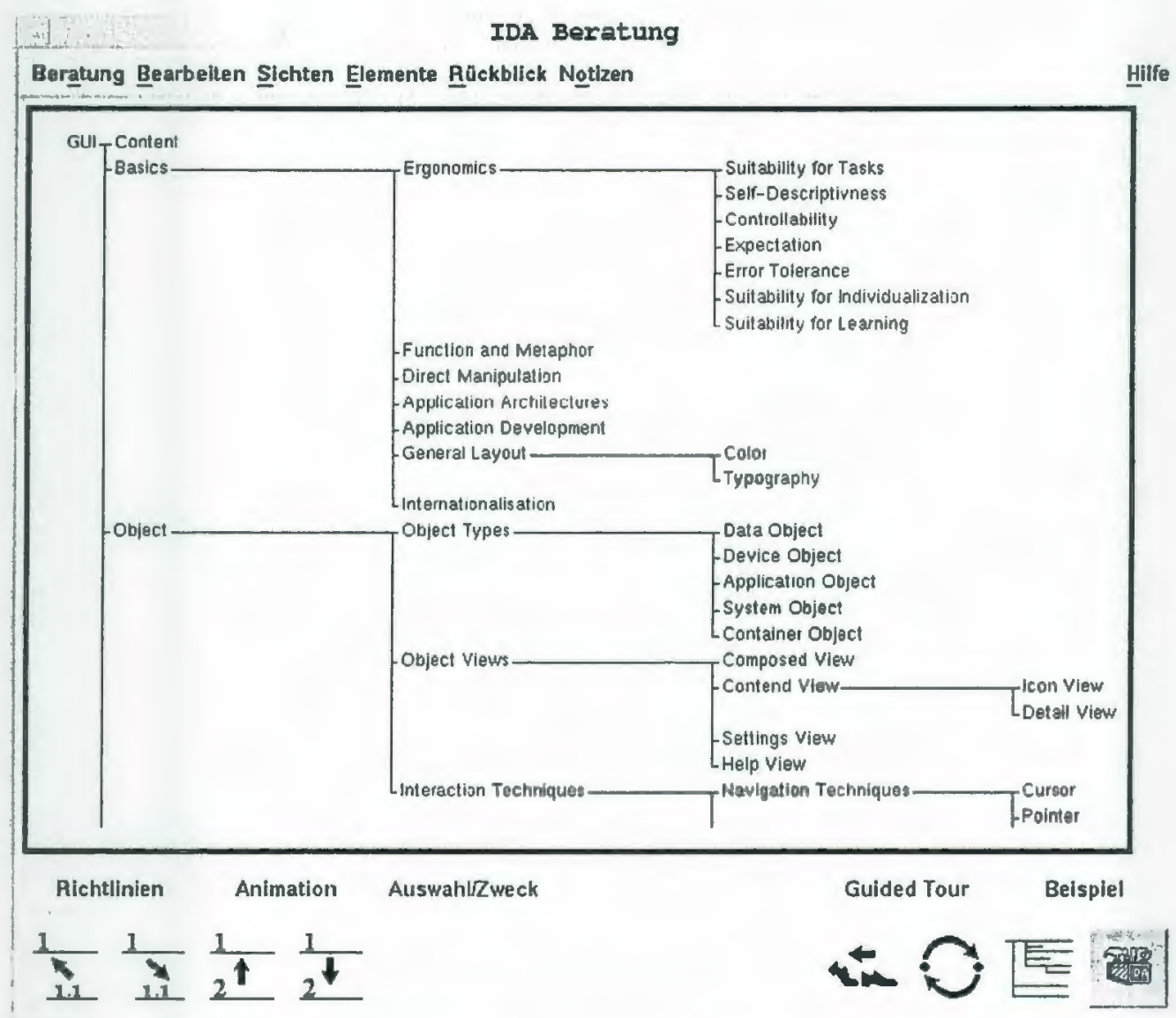

Abb. 2: Ausschnift aus dem Inhadtsverzeichnis der IDA-Beratung

Die Inhaltsstruktur wird in allen Komponenten der IDA-Entwicklungsumgebung in gleicher Weise reprïsentiert (z.B. Strukturierung der Inhalte der Klassenbibliothek, Strukturierung der Wissenshasis der Qualitiatssicherung), um eine globule Orientierung des Entwicklers zu unterstiitzen. Gleichzeitig kunn die Struktur der Querverweise in konventionellen Büchem auf die Hypertext-Links zwischen den einzelnen Beratungsthemen ahgebildet werden. Dies liefert eine zusätzliche, netzwerkartige Struktur, die die hierarchische Struktur iiherlagert. Als Problem bei dieser Alt der Strukturierung der Inhalte hat sich die Partitionierung in einzelne Beratungsthemen herausgestellt. Bei dieser vom Hypertext-Konzept geforderten Unterteilung stellte sich häufig das Problem 
zu entscheiden, wann eine weitere Differenzierung von einzelnen Beratungsthemen notwendig ist. Außerdem hat sich eine eindeutige Zuordnung von Beratungsthemen zu hestimmten Positionen in der hierarchischen Struktur als sehr schwierig erwiesen. Diese Probleme resultieren auch aus fortwährenden Weiterentwicklung des Ergonomiewissens und verlangen eine permanente Anpassung der Struktur.

\subsection{Präsentation der Inhalte}

Bei der Präsentation der Inhalte der IDA-Beratung an der Benutzungsoberflïche hat sich die Verwendung von Metaphern als niitzlich erwiesen [16]. Aufgrund der Konkurrenz zu schriftlichen Richtliniensammlungen bot sich die Wahl der Buch- bzw. RingbuchMetapher an. Die Implementierung der IDA-Beratung unter OSF/Motif, mit der Bezeichnung Multimedia Interface Design Documents for Advice (MIDA), verwendet die Buch-Metapher [2]. Für die Realisierung der IDA-Beratung unter Microsoft Windows, mit der Bezeichnung Interlace Design Advice Tool (IDAT), fiel die Entscheidung auf die eng verwandte Ringbuch-Metapher [16]. Diese unterstützt eine speziellen Explorationstechnik. Danach werden beim Verfolgen von Querverweisen die Seiten, die Ausgangspunkt von Querverweisen sind, aus dem Ringbuch entnommen und auf einen separaten Stapel abgelegt. Mit Hilfe der Seiten auf diesem Stapel kann der Entwickler dann stets die Eckpunkte seines aktuellen Explorationspfades rekonstruieren. Ziel dieser Technik ist eine bessere Orientierungsunterstitzung bei der Navigation durch den Themenraum. Im Rahmen der in Durchfïihrung betindlichen empirischen Evaluation soll die Eigung der verschiedenen Metaphern für Zwecke der Wissensvermittlung untersucht werden.

Abb. 3 zeigt eine typische Benutzungssituation der IDAT-Beratung. Der Stapel der durch das Verfolgen von Querverweisen herausgelegten Seiten wird dort durch die zurückliegenden und bis auf die Titelleiste verdeckten Fenster reprïsentiert (OverlappedWindows-Technik). Innerhalb eines Fensters erfolgt die Präsentation einer Seite auf fünf Karteikarten, die durch Klicken auf die zugehörigen Karteikartenreiter in den Vordergrund.gebracht werden können. Bei der IDAT-Beratung wurde eine strikte Komplexitätsreduzierung verfolgt [5]. Ziel war es, im Sinne einer kognitiv tieferen Informationsverarbeitung die Aufmerksamkeit des Entwicklers auf den Inhalt und nicht die Interaktionselemente und -techniken zu richten. Daher hefinden sich in den Themenfenstem der IDAT-Beratung keine Pull-Down-Menïs oder Push-Buttons. Die gesamte Funktionalität verbirgt sich in einem Pop-Up-Menü, das durch Drücken der rechten Maustaste aktiviert wird (siehe Abh. 3 Mitte). Zusiatzlich werden die wichtigsten Navigationsfunktionen auch in einer Toolbar angeboten (siehe Abb. 3 links oben). Eine ausschließBliche Bedienung per Tastatur ist ebenfalls möglich. Das Funktionalitätsangebot basiert auf Ergebnissen einer Befragung von Entwicklern der Software AG [6]. 


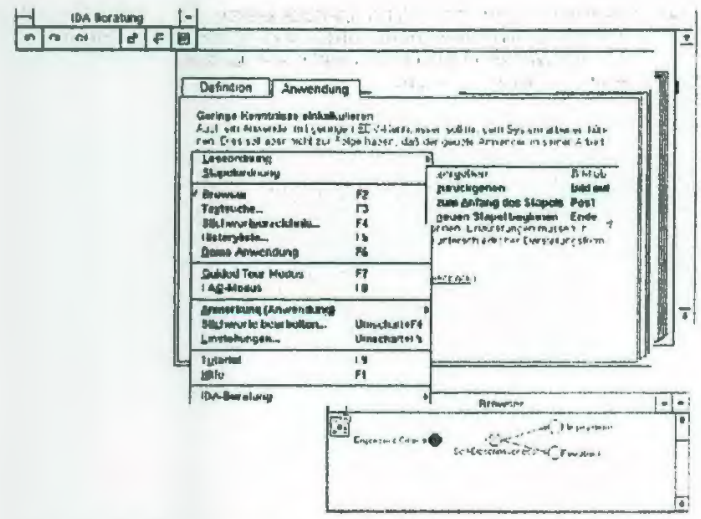

Abb. 3: Typische Benutzungssituation der II)AT-Beratung unter Windews

Im Vergleich dazu liegt dem MIDABeratungssystem die Buch-Metapher" zugrunde. Abb. 4 zeigt das MIDAHauptfenster, in dem auch ein graphischer Browser anzeigt werden kamn. Damit der Entwickler nicht mit zuviel Inhalten konfrontiert wird, wird der Inhalt in Teile gegliedert. Es wird unterschieden zwischen allgemeinen Erklärungen, sowie Richtlinien. Die allgemeinen Erkläiungen werden permanent im Hauptfenster angezeigt und geben eine kurze Information zum Thema an. Verwandte Themen und Abweichungen in den verschiedenen Style Guides werden ebentalls im Hauptfenster angezeigt. Die Richtlinien werden in einem eigenen Fenster präsentiert (Abb. 4 rechts oben). Der Entwickler kann die angezeigten Richtlinien interaktiv explorieren, indem er mit der Maus in die Beispielgraphik klickt. Zur Unterscheidung der Quellen der verschiedenen Richtlinien werden diese farbig angezeigt. So wird auch die besondere Wichtigkeit einer Richtlinie visualisiert (Muß/Kann). Bei Beratungsthemen, bei denen eine textuelle und graphische Priasentation nicht ausreicht, beispielsweise bei Dialogabläufen, wird das Beratungsthema animiert hzw. als Videosequenz aufgezeichnet und vertont. Diese Animationen können dann vom Entwickler, wie von einem Videorecorder bekannt, abgespielt, angehalten und fortgesetzt werden. 


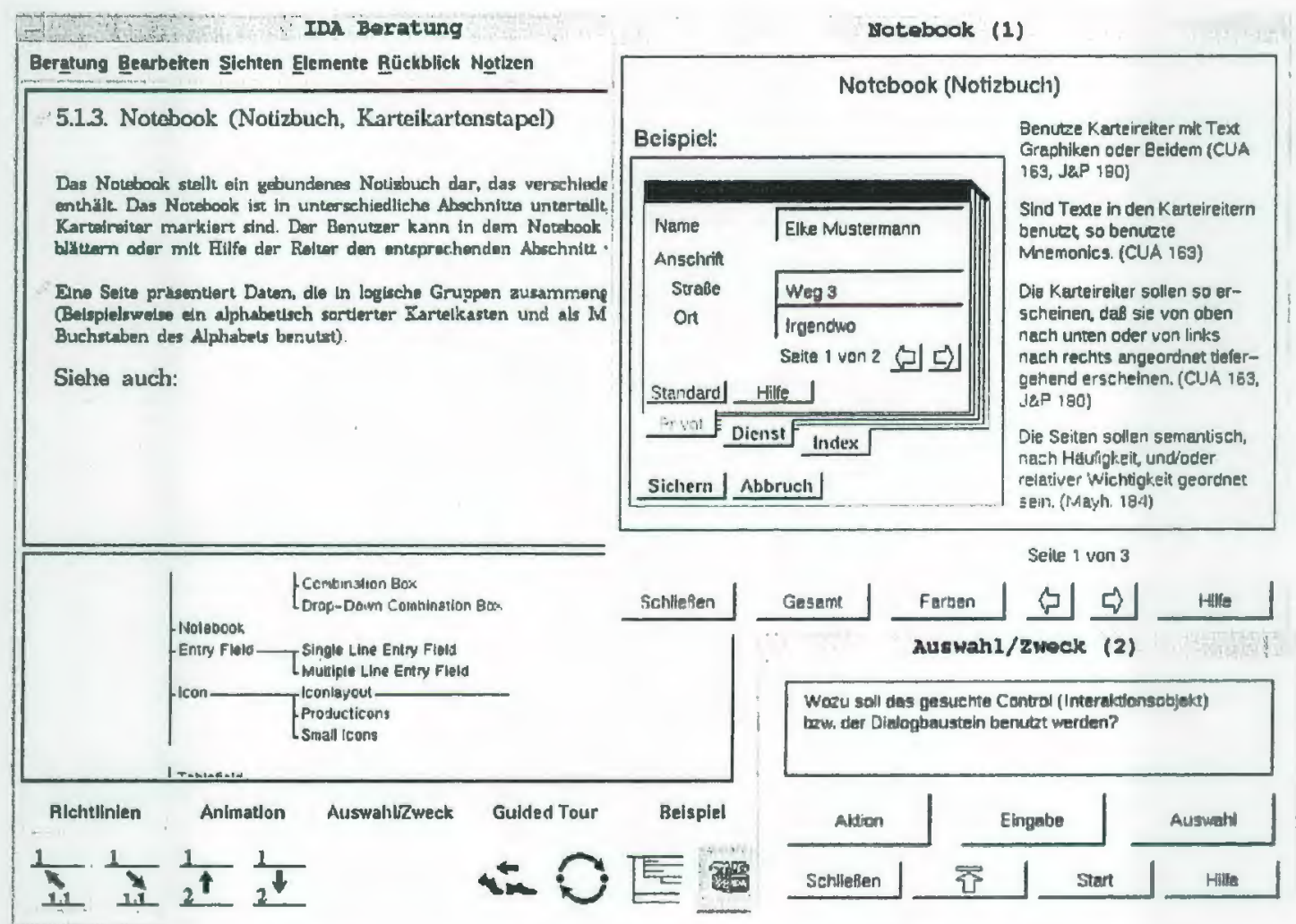

Abb. 4: Komponenten der MII)A-Beratung unter (JSF/Motif

\subsection{Navigationstechniken}

Neben bekannten Navigationsfunktionen hypermedialer Systeme, wie z. B. Hotlinks, Historylisten, Inhalts- und Stichwortverzeichnisse können Auswahldialoge als zusätzliche Navigationsmöglichkeit genutzt werden. Abb. 4 zeigt ein Beispiel des Auswahldialngs (Fenster Auswahl/Zweck), der dem Entwickler bei der richtigen Auswahl von Dialogobjekten unterstützt. Der Entwickler wird zunächst gefragt, ob das Dialogobjekt zur Eingahe, Auswahl oder Aktion dienen soll. Es werden anschliel.end weitere Fragen gestellt, bis zum Schlulß ein Beratungsthema zum vorgeschlagenen Dialogobjekt im Hauptfenster des Beratungssystems angezeigt wird.

Eine mehr themenübergreifende Sicht aul die Inhalte lielert die Guided Tour. Sie beschreibt einen vordefinierten Weg durch das Beratungssystem und erlaubt damit eine themenübergreifende Präsentation dessen Inhalte. 


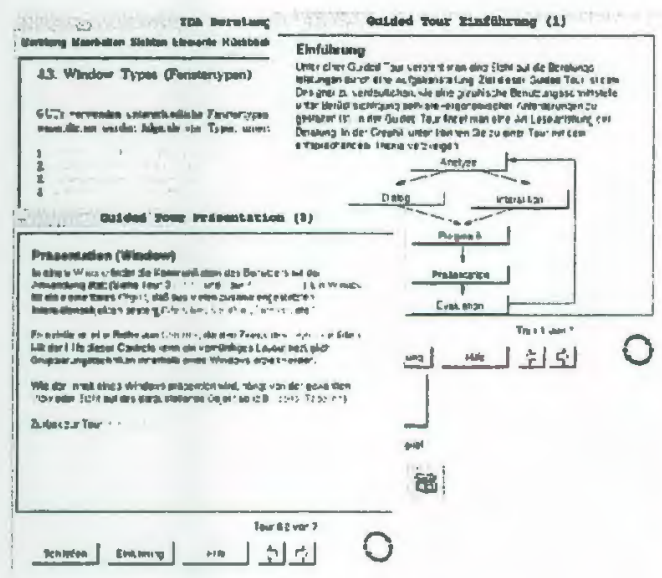

Abb. 5: Givided Tour der MIDA-Beratung unter OSF/Motif

Anhand der Guided Tour wird ein kompletter Oberflüchendesignzyklus erklïrt (Ahb. 5 zeigt das Startfenster rechts ohen). Deshalh wendet sich die Guided Tour vor allem an Anfänger im ergonomischen Ohertlïchendesign. Das Durcharbeiten eines bestimmten Abschnittes der Guided Tour erfolgt durch Aktivierung der gewiinschten Phase des Designzyklus. Wird beispielsweise die Phase Präsentation in Startfenster ausgewiahlt, so werden in einem neuen Fenster (Abb. 5 vorne), die durchzuführenden Aktivitïten heschriehen. Anhand von verschiedenfarbigen Hotlinks kann die Navigation beeinflulst werden. Wird auf einen grünen Hotlink geklickt, verzweigt der Entwickler innerhalb der Guided Tour. Wird hingegen ein blauer Hotlink ausgewaihlt, so wird das entsprechende Beratungsthema im Hauptfenster der Beratung (Abb. 5 im Hintergrund), angezeigt. Die gesamte Funktionalitait des Beratungssystems wird weiterhin zur Verfïgung gestellt, so daßs eine parallele Nutzung der Beratung und Guided Tour möglich ist.

\section{Verwandte Forschungsansätze}

Eine Auswertung einschlảgiger Forschungsvorhahen hat gezeigt, dal.s die Entwicklung von Unterstiitzungsleistungen für Entwickler von Benutzungsohertlächen bereits Gegenstand von zahlreichen Forschungsvorhahen war und ist.

Im Department of Computer Science der University of Colorado in Boulder werden seit einigen Jahren sogenannte Kritiksysteme entwickelt. Vor allem im Bereich Designunterstützung wurden eine Reihe von prototypischen Systeme entwickelt, die unter Verwendung von wissenshasierten Techniken, Hypertextsystemen und Bibliotheken wiederverwendbarer Designergebnisse dem Designer Unterstiitzung hieten [9].

Am Deparment of Computer and Information Science der University of Linköping wurde ein UIMS mit einem Expertensystem verbunden, das mittels einer Wissenshasis die entworfenen graphischen Benutzungsoherflächen nach Style Guide spezifischen Anforderungen analysiert. Ergebnis des Qualitätssicherungsprozesses sind Kommentare, die der Entwickler anschliel.end herïcksichtigen kann. [15]

Am College of Computing des Gerorgia Institute of Technology in Atkinta wird an der automatischen Generierung von Benutzungsoherflächen gearbeitet. Basierend auf einem 
objekt-orientierten Datenmodell werden automatisch - unter Beachtung von Style Guide spezifischen Anforderungen - Benutzungsobertlächen generiert und mittels eines UIMS visualisiert [1].

Am Fraunhofer-Institut für Arbeitswirtschaft und Organisation in Stutrgart wurde ebenfalls an der automatischen Generierung von Benutzungsoberflächen gearbeitet. Basierend auf einem Entity-Relationsship-Datenmodell, ergänzt um sogenannte Aufgabensichten und Dialognetzen, wurde mittels eines regelbasierten Systems automatisch die erste Version der Benutzungsobertläche - unter Verwendung von software-ergonomischen Designregeln - generiert und mittels eines UIMS visualisiert [13].

Im Fachbereich Informatik der Universitiit Oldenburg wird dem Entwickler von Benutzungsoberflächen ergonomisches Wișsen zur Verfügung gestellt. Anhand der Methode MUSE und eines wissenshasierten Beratungswerkzeuges soll sowohl Bewertungs- als auch Beratungsleistungen zur Verlïigung gestellt werden [10].

Am Lehrstuhl für Software-Technik der Ruhr-Universitiit Bochum wird aus einem objekt-orientierten Datenmodell mittels verschiedener wissenshasierter Generatoren, die auch Ergonomiewissen enthalten, automatisch eine Beschreibung einer Benutzungsoberfläche generiert. Diese wird dann mit Hilfe eines UIMS visualisiert und steht anschießend dem Entwickler zur weiteren Bearbeitung zur Vertïigung [3].

Am Institut fiir Angewandte Psychologie der Humbolt-Universitiat. Berlin wird im Rahmen eines Projektes ein Interface-Ratgeher entwickelt. Dieser soll sowohl als Textbuch [27] als auch als rechnerhasiertes Unterstiitzungswerkzeug verfüghar sein.

Im Fachbereich Informatik der Technischen Hochschule Darmstadt wird im Rahmen des Vorhabens Diades-II ein objekt-orientiertes Entwurl'swerkzeug, das auch Ergonomiewissen beinhaltet, für interaktive Benutzungsschnittstellen auf der Grundlage koagierender Agenten entwickelt [7].

\section{Resümee und Ausblick}

Die bisherigen Projektergebnisse zeigen, dal.s rechnerbasicrte Unterstïtzungswerkzeuge einen vielversprechenden Ansat\% \%ur Vermiltung und Umsetzung von soltware-ergonomischen Gestaltungsforderungen darstellen. Sie weisen somit einen möglichen Weg zur konsequenten Umsetzung von software-ergonomischen Gestaltungsforderungen, direkt im Implementierungsprozeß von graphischen Benutzungsoherflächen. Diese prospektive Gestaltungsorientierung ist ein entscheidender Vorteil dieses Ansatzes gegenüber der korrektiven Gestaltungsorientierung traditioneller software-ergonomischer Evaluationsverfahren (z.B. EVADIS, [24]). Entscheidend für die Akzeptanz derartiger Unterstützungswerkzeuge ist einerseits das Gelingen der Integration in das primäre Entwicklungswerkzeug des Entwicklers und andererseits das unmittelbare Auftinden 
und Anwenden von Wissensinhalten. Dies berührt Fragen von geeigneten Retrieval Strategien und Mechanismen (z.B. Browser, Suchfunktionen, Guided Tour) sowie von geeigneten Präsentationsformen von Wissensinhalten (z.B. objekt-orientiert, multimedial, wissenshasiert). Durch die Anwendung einer Vielzahl von Retrieval Strategien und Wissenspräsentationsformen im beschriebenen Vorhaben wurde vorerst versucht, einen möglichst maximalen Rahmen aufzuspannen.

Der nächste Schritt besteht in der Evaluation der verschiedenen Konzepte hinsichtlich Eignung und Akzeptanz. Die in Durchführung hefïndlichen empirischen Evaluationen der verschiedenen Unterstützungswerkzeuge bei den Industriepartner sollen unter anderem Antworten auf folgende Fragestellungen liefern: Eignung der einzelnen Unterstützungswerkzeuge für Zwecke der Konstruktionsunterstïtzung, der multimedialenBeratung und der automatisierten Qualitåtssicherung; Vorteile und Grenzen der Wissensprïsentation in den jeweiligen Unterstützungswerkzeugen; erzielbare synergetische Effekte durch das Ineinandergreifen der verschiedenen Unterstützungswerkzeuge; Akzeptanz der zusätzlich zum eigentlichen Entwicklungswerkzeug zu nutzenden Unterstützungswerkzeuge durch die Entwickler.

Basierend auf den Evaluationsergebnissen ist eine Reimplementierung der Unterstützungswerkzeuge geplant, die geeignete und akzeptierte Konzepte und Mechanismen beibehält bzw. vertieft und ungeeignete hzw. nicht akzeptierte verwirtt.

\section{Literatur}

[1] Baar, D., Foley, J., Mullet, K.: Coupling Application Design ind User Interfice Design. In:

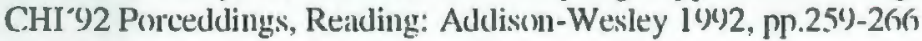

[2] Bachmann, F.: Entwicklung des multimedialen Beratungssystem MIDA zur Gestaltung von Benutzungsoberflichen unter software-ergonomischen Giesichtspunkten, Diplonarbeit, Universibitt Bonn, Bonn, 1994 .

[3] Balzert, H.: Der IANUIS-Dialogexperte: Vom Falchkonzept zur Dialogstruklur. Soltwaretechnik Trends, Bind 13, Heft 3, 10)3, \$.62-72

[4] Beimel, J., Hütmer, J. und Windke, H.: Kenntnisse von Progranmierern auf dem Giebiet der Software-Ergonomie: Stand und Möglichkeiten zur Verhesserung, unpublished paper of a lecture on the Fachtagung der Sektion Arbeits-, Betriebs und Organisallionspsychologie des Berufsvetbandes Deutscher Psychologen "Arbeits- Betriehs- und (Organisationspsychologie vor Ort" 25.-27.5.19)2, Bad Lauterbich.

[5] Büclıner, S.: Komplexilätsreduzienung der Benutzerobertlïche der IDA-Beratungs- und Erkliirungs-sebene, Ergonomie-Studienarbeit, Universitii/(Gesanthochschule Essen, 1994.

[6] Diehl, U.: Entwicklung einer Online-Beratung zur ergonomischen Gestaltung von Windows Anwendungen - demonstriert an Beispiel der Entire office Workstation cher Soltware AG, Diplomarbe it, Fachhochschule Daunstidt, Darmstadt, 1964. 
[7] Dilli, I., Hoffmamn, H.-J., Koschorek, D.: Experience with an object-oriented design approach for at progranıning environment under a blackboard architecture. In: Lisker C.E. (ed.) Advances in Human Systems and Information Technology, The Intemational Institute for Advinced Studies in Systems Research and Cybernetics 1\%92, pp.268-276

[8] Europäische Giemeinschatt: Richllinie über die Mindestvorschniften hezüglich der Sicherheit und des Gesundheitsschutzes bei der Arbeit in Bildschinngeriten (EWG 9()/27()).

[9] Fischer, G., Nakakouji, K., ()stwald, J., Stalhl, G., Sumner, T.: Embedding Computer-Based Critics in the Contexts of Design. In: INTERCHI ')3 Procedings, Reidling: Addism-Wesley 1)(3, pp. $157-164$

[10] Gorny, P., u.a.: Projekt EXP()SE, Expertensystem "zur phasenorientictten Soltwaur-ErgonomieBeratung bei der Benutzungsschnittstellen-Entwicklung, 2. Zwischenbericht, Universitit Oldenburg und Universitït Rostock. 19(9)

[11] IBM: Object-Oriented Interfice Design, IBM Common User Access Guidelines. Carmel: Que Corporation. 1992.

[12] ISO 9241: Ergonomic Reguirements for Olfice Work with Visual Display Terminals.

[13] Janssen, C. , Weisbecker, A., Ziegler, J.: Generation User Interfaces form Datit Moxlels and Dialogue Net Specifications. In: INTER(HI ${ }^{`} 3$ Procedings, Readling: Addison-Wesley 19)3, pp. $418-423$

[14] Jenz \& Partner. Gratische Bediener-Ohertlichen, Erlensee, Jenz \& Partner, 1\%)2.

[15] Löwgren, J., Nondq̨uist, T.: Knowledgre-Bassed Evaluation as Design Support for Graphical User Interfaces. In: (.HI")2 Porceddings, Reading: Addlison-Wesley 19(2), pp. I81-188

[16] Mana, S., Oberquelle H.: Perspectives and Metaphors for Human-Computer Interaction. In Floyd, C., Züllighoven, H., Budkle, R., Keil-Slawik, R. (Hrsg.): Soffware-Developinent ind Reallity Construction, Berlin: Springer, 19)2, pp.233-251

[17] Mayhew, D.: Principles and Ciujdelines in Software User Interfice Design, Englewond C.liffs: Prentice Hall, l())2.

[18] Mircorsoft: The Windows Interfice, An Applicattion Design Guide, Microsoft Press, 1 (y)2.

[19] Molich, R., Nielsen, J.: lmproving a human-computer diadoguc. Comnunications of the ACM, vol. 33, no. 3, 19(), pp.338-348.

[20) Müller, M.: Erweiterung des Hyperlextsystems ToolBenk um Hillsmittel zur Reduzierung der Gefähr des Orientierungsverlustes, Diplomarbeit, Universitiit / Gesiunuhnchschule Padertxom, Fachbereich Mathematik / Infonmatik und Wirtschaftswissenschalten, Patderbom, 1943.

[21] Open Software Foundation: OSF/MOTIF Style Guide, Revision 1.2, London: Prentice-Hall, 1943.

[22] Porschen, M.: Hypermediale Beratungssysteme - Herleitung und Evaluation eines werkzeugunathhängigen und plattiormübergreifenden Entwicklungskonzeptes, Diplonauheit, Universität Bınn, Bonn, 1994.

[23] Raunn, M.: Generierung und Verwaltung einer Bibliothek von Modellvorlagen zur Gestaltung software-ergononnischer Benutzungsobernliichen, Diplomarbeit. Universitäi Bomn, Bonn, 1 (\%) . 
[24] Reiterer, H., Oppermann, R.: Evaluation of user interfaces, EVADIS II - a comprehensive evaluattion approach, Bchaviour \& Information Technology, Vol. 12, No. 3, pp.137-148.

[25] Reiterer, H.: A User Interface Design Assistant Approach, in: Brunnstein K., Raubold E. (eds.): Applications and Impacts, Information Processing '94. Proceedings of the IFIP 13th World Computer Congress, Hamburg, Germany, 28 August - 2 September, 19\%4, IFIP Transactions A52. Volume II, North-Holland, Amsterdam. 1994, pp. 180-187.

[26] Schäfer, S.: Qualitattsicherung im Isser Interfice Design, Chancen und (irenzen, Diplomarbeit, Universität Bonn, Bonn, 1994.

[27] Wandke, H., Hüttner, J., Rïtz, A., Vogler, K.: Broschüre des Informations und Beratungssystems

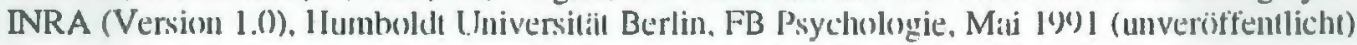

Frank Bachmann ${ }^{1}$, Michael Porschen ${ }^{1}$, Manfred Ramm ${ }^{1}$, Dr. Harald Reiterer ${ }^{1+2}$, Stefan Schäfer ${ }^{-1}$, Helmut Simm ${ }^{1}$

1 Gesellschaft für Mathematik und Datenverarbeitung (GMD)

Schloß3 Birlinghoven, D-53731 Sankt Augustin

2 Universität Wien, Institut für Angewandte Informatik und Informationssysteme Liebiggasse 3/3-4, A-1010) Wien 